\title{
Erodible, granular beds are fragile
}

\author{
Diego Berzi, ${ }^{1}$ James T. Jenkins, ${ }^{2}$ and Patrick Richard ${ }^{3}$ \\ ${ }^{1}$ Department of Civil and Environmental Engineering, Politecnico di Milano, 20133 Milano, Italy. \\ ${ }^{2}$ School of Civil and Environmental Engineering, Cornell University, Ithaca, NY 14853 USA. \\ ${ }^{3}$ IFSTTAR, site de Nantes, GPEM/MAST, 44344 Bouguenais, France.
}

(Dated: August 30, 2019)

\begin{abstract}
Geophysical flows that involve the transport of grains and the shearing of colloids and nonBrownian suspensions often take place above a substrate composed of the same particles that can be incorporated into the flow. Despite the importance of understanding such erodible beds to the phrasing of appropriate boundary conditions for the solution of continuum models, a rigorous definition of the erodible bed and the constitutive relations for the stresses within it are still lacking. Here, we use discrete-element simulations to show that the intense, intermittent forming and breaking of contact chains marks the transition to the erodible bed at a critical solid volume fraction, as in shear jamming of steady, homogeneous flows. However, the compressible, collisional flow that confines the bed is not strong enough to insure the stability of the contact network, resulting in a bulk stiffness that is three orders of magnitude less than in shear jamming.
\end{abstract}

Many geophysical flows of interest in Nature, such as turbidity currents [1], Aeolian [2] and aquatic sediment transport [3], and debris flows [4], involve contact with a bed of solid particles that apparently, are immobile. Although it is now clear that the particles in the erodible bed creep [5,6], their contribution to the total transport is almost always negligible. However, mathematical continuum models of geophysical flows require boundary conditions at the interface between the flow and the bed and the quantification of the mass and momentum exchange across it $[7,8]$. In this regard, a better understanding of the physics that governs the erodible beds is crucial.

To obtain this here, we make use of discrete element simulations of inelastic, frictional spheres in steady, unidirectional flows, both homogeneous and inhomogeneous (Figs. 1a-e). In these, the particles interact thorough linear springs and dashpots in both the normal and tangential directions. Particles are either identical or slightly polydispersed spheres with an average diameter $d$, maximum polydispersity equal to $20 \%$, and mass density $\rho_{p}$, characterized by a coefficient of normal collisional restitution $e$ and sliding Coulomb friction $\mu$. The stiffness of the linear normal spring in the interaction model is $k$. See the Supplementary Information for more details about the numerical simulations.

In homogeneous, steady shearing flows, in which either the volume or pressure is imposed (Figs. 1a and b), the solid volume fraction, $\nu$, is uniform over the domain. These flows are then commonly used to infer information about the local rheological behaviour of granular materials $[10,11]$, to be applied in more complex situations. When gravitational acceleration, $g$, and/or lateral confinement are present, inhomogeneity in the distribution of solid volume fraction develops. Then, we may distinguish between a region in which the particles mainly interact through collisions and the shear rate is significant, the flow, and a region where contacts are enduring and the shear rate is exponentially decaying, the bed. Inhomogeneity in the presence of a bed typically occurs in steady and fully developed pressure-imposed shear flows (Fig. 1c), chute flows (Fig. 1d) and inclined, free surface flows between frictional sidewalls (Fig. 1e).

The identification of the location of the interface between the flow and the bed is, however, challenging. In the case of inclined, free surface flows, for example, the profiles of the mean velocity, $u$, solid volume fraction and average number of contacts per particle (the coordination number), $Z$, are continuous, with no obvious signature of a transition to the bed (Figs. 1f-h). (author?) [6] take the interface between the flow and the erodible bed to be where the ratio of the shear stress, $s$, to the pressure, $p$, reaches a characteristic value associated with yielding. This value for the stress ratio has been largely adopted as a boundary condition for flows over erodible beds [1215]. (author?) [6], however, noted that the solid volume fraction also reaches a characteristic value at the interface with the erodible bed. The existence of a particular solid volume fraction, marking the transition from flow to bed, hints at the role played by the configurations of the grains and the space available for their motion.

Existing simulations of volume-imposed simple shearing [10] show that rate-independent components of the stresses are present only above a critical volume fraction, $\nu_{c}$, which is a decreasing function of the coefficient of sliding friction. Because there is one-to-one relation between solid volume fraction and coordination number in simple shearing [16], a critical coordination number, $Z_{c}$, also exists. Both $\nu_{c}$ and $Z_{c}$ are independent of the particle stiffness $[17,18]$. Given that $Z_{c}$ is greater than four for all values of coefficient of sliding friction [16], the simple shearing flows with $\nu \geq \nu_{c}$ can be identified as mechanically stable, shear-jammed states [19]. (author?) [11] modelled these as networks of springs, in which the nodes are continuously changing due to the particle rearrangement, with the springs, on average, compressed, 


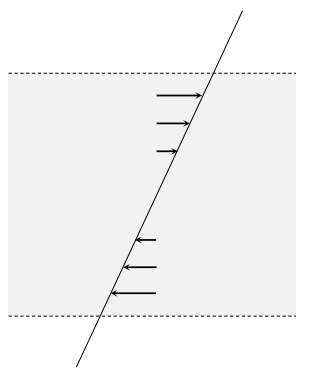

(a)

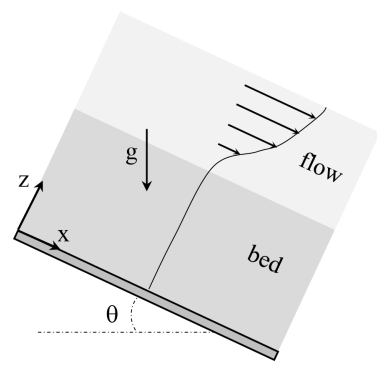

(e)

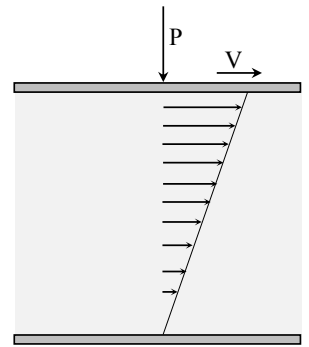

(b)

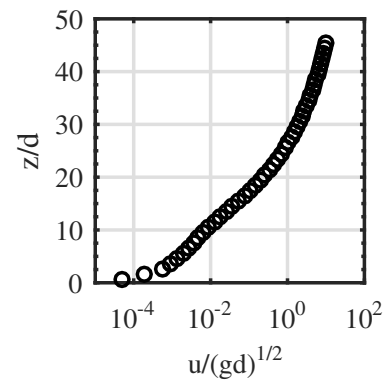

(f)

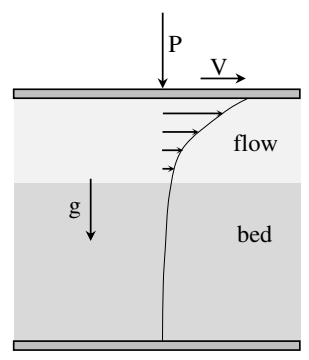

(c)

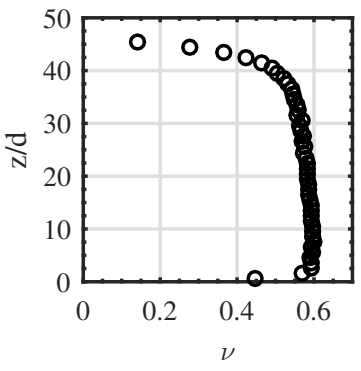

(g)

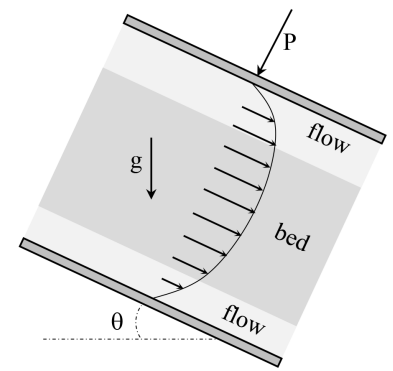

(d)

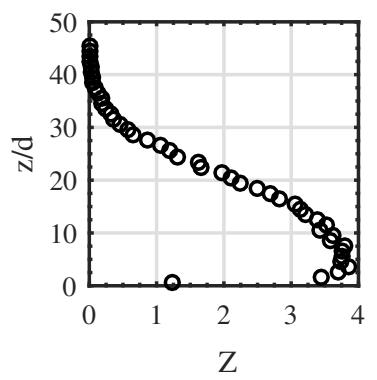

(h)

Figure 1. Configurations and qualitative profiles of mean velocity in the flow direction: (a) volume-imposed simple shear flow in a periodic cell [9]; (b), (c) pressure-imposed shear flow in the absence and presence of gravity, respectively; (d) pressure-imposed chute flow; (e) inclined, free surface flow between frictional sidewalls. Profiles of: (f) dimensionless mean velocity, (g) solid volume fraction and (h) coordination number, shown in the case of an inclined, free surface flow, with $\theta=30^{\circ}$ and the sidewalls 30 diameters apart.

due to the particle overlaps. The spring compression is responsible for the rate-independent components to the stresses and their proportionality to the particle stiffness, while the fluctuations in the node velocity and the associated momentum exchange is responsible for the rate dependence.

Three methods have been proposed to identify the critical volume fraction in discrete element simulations of simple shearing. First, by measuring the ratio of contact duration to passage time in simple shear over a range of volume fractions and plotting curves of coordination number versus volume fraction for different values of the ratio. Such curves intersect at the critical point, providing both $\nu_{c}$ and $Z_{c}[17,18]$. Second, by plotting the dimensionless pressure $p d / k$ as a function of the time ratio for different values of the solid volume fraction. The minimum value of the solid volume fraction for which $p d / k$ becomes independent of the time ratio, as the ratio approaches zero, is $\nu_{c}[10]$ - that is, at which the pressure in the rigid limit is rate-independent. Third, by taking advantage of the large fluctuations in coordination number and pressure observed near $\nu_{c}$, as the network of contacts intermittently forms and breaks [10]. A plot of the standard deviation of the fluctuations in coordination versus volume fraction shows a peak at $\nu_{c}$. Only this third criterion can be easily applied to inhomogeneous flows.

The temporal evolution of the coordination number at

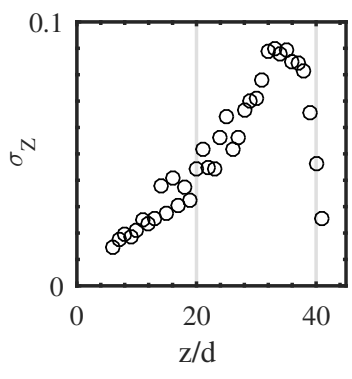

(a)

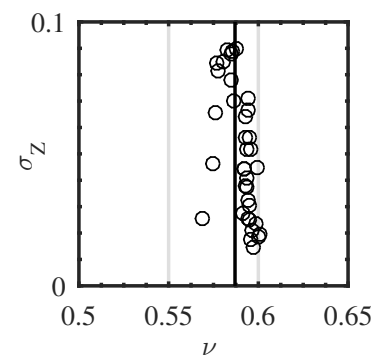

(b)
Figure 2. Inclined, free surface flow between frictional sidewalls with $\theta=24^{\circ}$ and sidewalls 30 diameters apart: standard deviation of the fluctuations in the coordination number as a function of (a) elevation and (b) local, mean solid volume fraction. The solid, vertical line in Fig. $2 \mathrm{~b}$ is at $\nu=0.587$, the value of $\nu_{c}$ when $\mu=0.5[10]$.

different elevations above the rigid base for the inclined, free surface flows shows that the fluctuations in coordination number are maximum at a particular elevation above the rigid base. Assuming that the peak in the local standard deviation of $Z, \sigma_{Z}$, is a signature of the intermittent breaking and forming of contacts at the interface between the flow and the bed, the location of the latter can, therefore, be identified (Fig. 2a). A plot of $\sigma_{Z}$ as a function of 
$\nu$ (Fig. 2b) permits the identification of the solid volume fraction that marks the transition from the flow to the bed. This solid volume fraction is remarkably close to the critical value 0.587 identified in volume-imposed simple shearing of frictional particles for the same sliding friction, $\mu=0.5[10]$. This suggests that the transition from flow to bed is where the solid volume fraction reaches the critical value at which rate-independent components of the stresses arise in homogeneous flows. Consequently, it is reasonable to apply constitutive relations that successfully describe the stresses in volume-imposed simple shearing when $\nu \geq \nu_{c}$ [11] to the particles in the erodible bed.

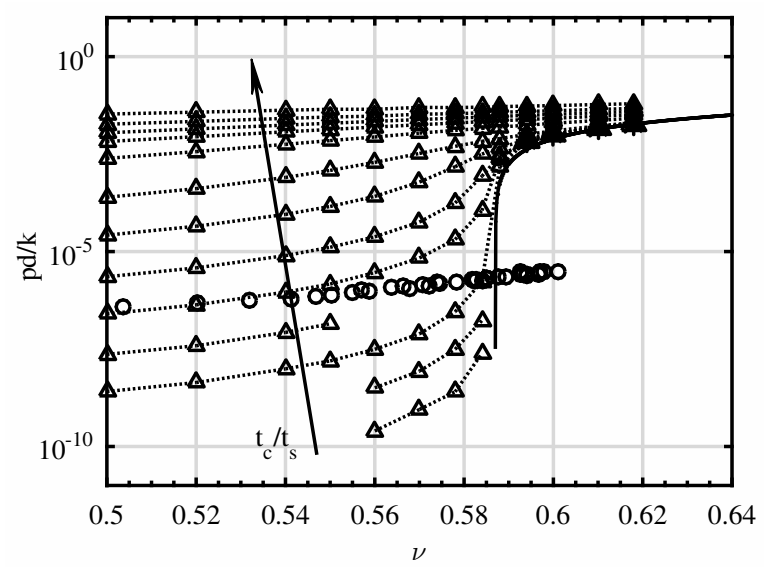

Figure 3. Dimensionless pressure $p d / k$ as a function of solid volume fraction in simulations of volume-imposed simple shearing [10] (triangles) at different values of $t_{c} / t_{s}$ and in simulations of inclined, free surface flows (circles) with $\theta=30^{\circ}$ and sidewalls 30 diameters apart. The solid line is the rateindependent component in Eq. (1).

(author?) [11] express the pressure for volume fractions above the critical volume fraction as

$$
\frac{p d}{k}=\frac{5 \nu(1+e)}{6}\left(\frac{\rho_{p} T d}{k}\right)^{1 / 2}+0.6\left(\nu-\nu_{c}\right),
$$

where the first term on the right-hand side is the ratedependent component associated with momentum exchange in collisions, with the frequency of collisions inversely proportional to the contact duration; and the second is the rate-independent component. The rateindependent component is the lower limit for the pressure, when $\nu$ exceeds $\nu_{c}$, as confirmed in the volumeimposed simple shearing simulations of (author?) [10]. However, in the inclined, free surface flows, the pressure for volume fractions above the critical is orders of magnitude less than that predicted by Eq. 1 (Fig. 3).

In an attempt to understand this, we performed volume-imposed simple shearing simulations of particles with the same coefficients of restitution and sliding friction as in the inclined. free surface flows. The simulations

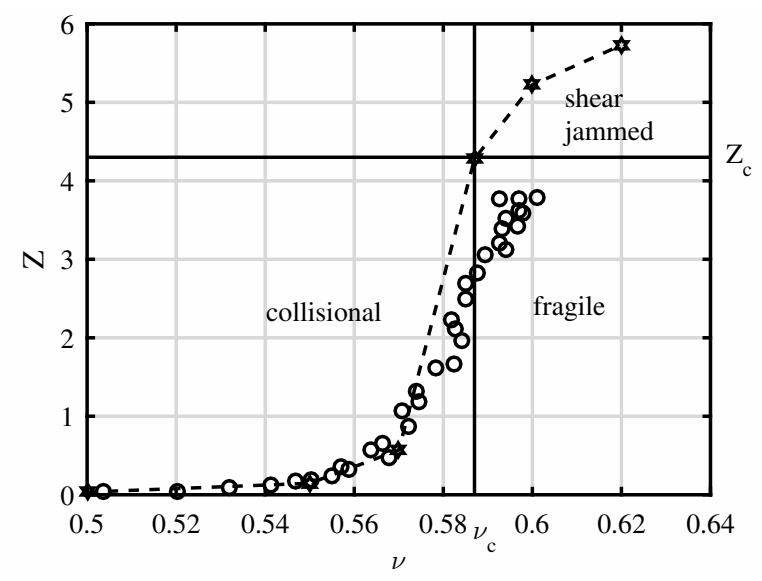

Figure 4. Coordination number as a function of solid volume fraction in our volume-imposed simple shearing (six-pointed stars) and in our inclined, free surface flows (circles) with $\theta=30^{\circ}$ and sidewalls 30 diameters apart. The vertical and horizontal solid lines identify the critical point, $\nu_{c}=0.587$ [10] and $Z_{c}=4.3$ [16], for shear jamming when $\mu=0.5$.

were carried out at different values of the solid volume fraction, matching the corresponding values of the ratio of the contact duration, $t_{c}=\left(\rho_{p} d^{3} / k\right)^{1 / 2}$, over the passage time, $t_{s}=1 / u^{\prime}$, where $u^{\prime}$ is the shear rate, measured in the inclined flow. A plot of the coordination number versus the solid volume fraction (Fig. 4) highlights two key points.

First, for $\nu \leq \nu_{c}$, the coordination number is less than unity, both in volume-imposed simple shearing and in inclined, free surface flows, even for volume fractions as large as 0.57. That is, in the absence of a network of contacts, particle interact essentially through binary, nearly instantaneous collisions, thus justifying the use of kinetic theory of granular gases, even for very dense flows [20].

Second, when $\nu$ exceeds $\nu_{c}$, the contact network in volume-imposed simple shearing is mechanically stable, given that $Z \geq Z_{c} \geq 4$. The use of periodic boundary conditions permits the contact network to extend indefinitely in the domain, and shearing requires significant compression of the particles, thus generating enormous stresses. In inclined, free surface flows, however, $\nu_{c}$ can be exceeded at values of the coordination number less than $Z_{c}$. The contact network is not then mechanically stable and particle rearrangement is possible without a dramatical increase in the stresses. Therefore, we distinguish between a shear-jammed state, when $\nu \geq \nu_{c}$ and $Z \geq Z_{c}$, and a fragile state, when $\nu \geq \nu_{c}$ and $Z<Z_{c}$.

It has been shown for volume-imposed simple shearing of frictional particles [10] that when the dimensionless pressure, $p d / k$, and the dimensionless shear rate, $u^{\prime} /\left[k / \rho_{p} / d^{3}\right]^{1 / 2}$, equal to the ratio $t_{c} / t_{s}$, are scaled with some powers of the distance to jamming, $\left|\nu-\nu_{c}\right|$, the relation between pressure and shear rate can be repre- 


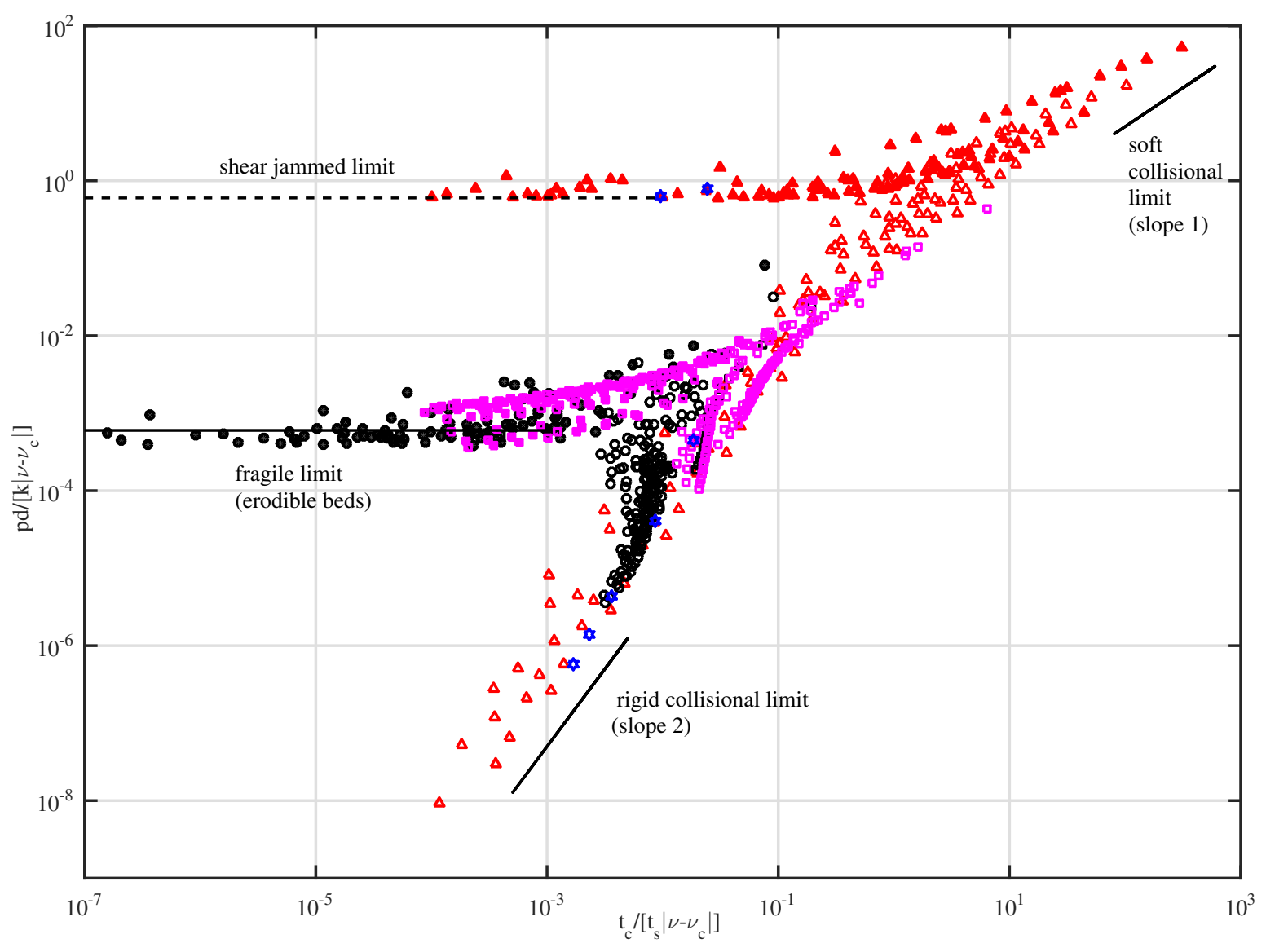

Figure 5. Scaled pressure versus scaled time ratio for: simulations of volume-imposed simple shearing [10] (red triangles) and our work (blue six-pointed stars); simulations of pressure-imposed simple shearing, shear flows and chute flows [21] (magenta squares); simulations of inclined, free surface flows (black circles). Open and filled symbols refer to volume fractions less than and greater than the critical, respectively.

sented by two universal curves, one for $\nu<\nu_{c}$ and one for $\nu \geq \nu_{c}$. This is compatible with the theory of (author?) [11], in which it is suggested that the exponent of the distance to jamming should be unity in both the scaled pressure and shear rate. Indeed, in simple shearing, the fluctuation energy produced by the working of the shear stress balances the rate at which fluctuation energy is dissipated in collisions, so that the granular temperature $T$ is proportional to $\left(u^{\prime} d\right)^{2}$, through a function of the solid volume fraction that remains finite at $\nu=\nu_{c}[22]$.

With this, it is straightforward to show that Eq. 1 can be rewritten as a relation between the scaled pressure $p d /\left(k\left|\nu-\nu_{c}\right|\right)$ and the scaled time ratio $t_{c} /\left(t_{s}\left|\nu-\nu_{c}\right|\right)$ with two asymptotes. The first is when $t_{c} /\left(t_{s}\left|\nu-\nu_{c}\right|\right)$ goes to zero (the rigid limit), so that $p d /\left(k\left|\nu-\nu_{c}\right|\right)$ is proportional to $\left[t_{c} /\left(t_{s}\left|\nu-\nu_{c}\right|\right)\right]^{0}$; that is, the pressure originates from the persistent compressions of the springs in the contact network and is rate-independent (the shear-jammed regime). The second is when $t_{c} /\left(t_{s}\left|\nu-\nu_{c}\right|\right)$ goes to infinity (the soft limit), so that $p d /\left(k\left|\nu-\nu_{c}\right|\right)$ is proportional to $\left[t_{c} /\left(t_{s}\left|\nu-\nu_{c}\right|\right)\right]^{1}$; that is, the pressure originates from momentum exchange during particle rearrangement with the frequency of interaction equal to the inverse of the contact duration (the soft collisional regime). The latter asymptotic regime is also shared by particle flows at $\nu<\nu_{c}$, when $t_{c} /\left(t_{s}\left|\nu-\nu_{c}\right|\right)$ goes to infinity, showing little to no dependence on the solid volume fraction [11].

In the rigid limit, when $\nu$ is larger than 0.5 , but less than $\nu_{c}$, the pressure is given by the classic expression of kinetic theory, $p \propto \rho_{p} T /\left(\nu_{c}-\nu\right)$, where the dependence on the solid volume fraction results from the divergence of the probability of having two particles in contact as $\nu_{c}$ is approached [23]. In simple shearing, this corresponds to a third asymptotic regime, that is $p d /\left(k\left|\nu-\nu_{c}\right|\right)$ proportional to $\left[t_{c} /\left(t_{s}\left|\nu-\nu_{c}\right|\right)\right]^{2}$, when $t_{c} /\left(t_{s}\left|\nu-\nu_{c}\right|\right)$ goes to zero (the rigid collisional regime). All the data from the simulations considered in the present work are shown in Fig. 5, in terms of dimensionless pressure against dimensionless shear rate.

A fourth, asymptotic, rate-independent regime, that 
we identify as the fragile regime, is clearly visible. Data in this regime belong only to pressure-imposed shear, chute and inclined, free surface flow simulations, where a region in which $\nu \geq \nu_{c}$ (the erodible bed) is in contact with at least one region in which $\nu<\nu_{c}$ and the mean interparticle distance is greater than zero. The collapse of the data coming from different configurations and sources is suggestive of a universal behaviour of the grains in the erodible bed.

There are two reasons for the scatter of the data in Fig. 5. First, there is a residual dependence on the solid volume fraction in the relation between the granular temperature and the shear rate, even in simple shearing, that is not completely captured in terms of powers of $\left|\nu-\nu_{c}\right|$. Second, in inhomogeneous flows, the shear rate and the granular temperature are related through a differential, not an algebraic, equation. The granular temperature is a more fundamental variable and should be employed instead of the shear rate. However, some datasets do not include the granular temperature, but only the shear rate. Also, the granular temperature is very sensitive to the size of the cells employed for coarse graining the measurements in the simulations [24], thus adding another source of scatter.

It is also worth mentioning that other authors [10] have employed different powers of the distance to jamming to scale the dimensionless pressure and shear rate. This has no consequence, however, on the slopes associated with the shear-jammed, fragile and rigid collisional limits in Fig. 5 and, therefore, on our findings about the nature of the erodible beds. Only the slope of the soft collisional limit is affected by the exponents of $\left|\nu-\nu_{c}\right|$, because the pressure there is independent of the solid volume fraction, at least in dense situations.
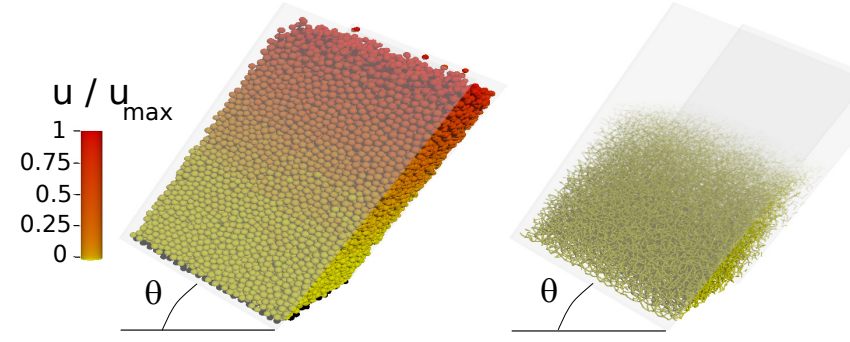

Figure 6. Snapshots of particle positions (left, coloured based on velocity intensity, with $u_{\max }$ maximum velocity in the flow direction) and force chains (right) in an inclined, free surface flow, with $\theta=30^{\circ}$ and the sidewalls 30 diameters apart. There are three flow regimes: collisional flow with near-instantaneous, uncorrelated, binary collisions well above the bed; collisional flow closer to the bed with correlated collisions and enough overlaps to produce short force chains; and a mixture of collisions and contacts within the bed that generated force chains that extend to the base of the flow.

We contend that the difference between the shearjammed of volume-imposed simple shearing and the frag- ile state of inclined, free surface flows in Figs. 4 and 5 is due to the fact that the contact network in the former extends indefinitely, because of the use of periodic boundary conditions, thus making it mechanically stable. In contrast, in an inclined, free surface flow, the top of the erodible bed is in contact with a collisional region that can be easily compressed, thus facilitating the buckling of the contact chains in the erodible bed, leading to mechanical instability and low coordination number (Fig. 6).

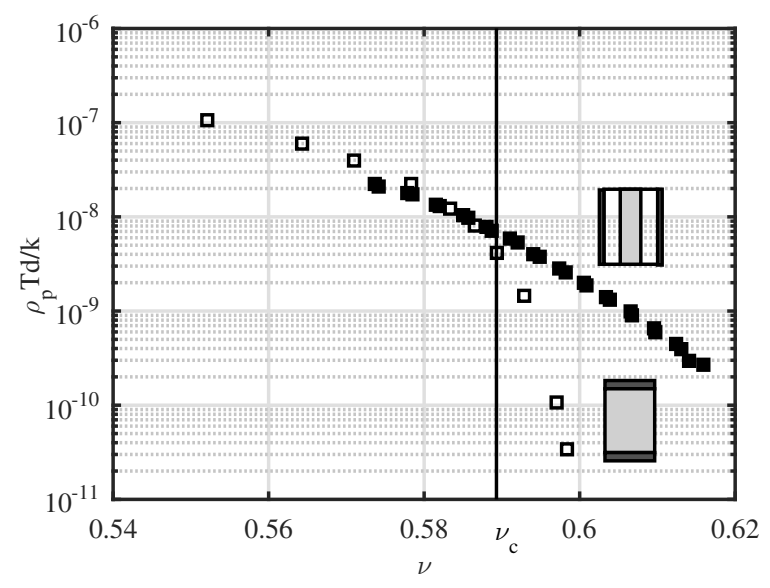

Figure 7. Dimensionless granular temperature as a function of solid volume fraction for pressure-impose simple shearing (open squares) and vertical chute flow (filled squares) simulations, with $p d / k=5 \cdot 10^{-6}[21,25]$. The solid line is at $\nu=0.589$, the value of $\nu_{c}$ when $\mu=0.4$ [16]. The gray region in the sketches of the two configurations is the region in which $\nu$ exceeds the critical.

The role of the boundaries can be assessed by performing, for example, simulations of pressure-imposed simple shearing and vertical chute flows, in which the dimensionless pressure, $p d / k$, is kept constant [25]. In both configurations, the flow is confined between two rigid, rough boundaries that enforce a no-slip boundary condition. In the pressure-imposed simple shearing, the solid volume fraction is uniformly distributed, so that once the volume fraction exceeds the critical, it does that everywhere. In this case, the contact network spans the entire domain and both ends of the contact chains are in contact with solid boundaries. In the vertical chute flow, instead, the critical volume fraction is exceeded only in a core region that is bounded by two compressible, collisional flows (inset of Fig. 7). When $p d / k$ is constant, the equation of state (Eq. 1) reduces to a universal relation between the dimensionless granular temperature, $\rho_{p} d T / k$, and the solid volume fraction, at least in simple shearing [11].

Fig. 7 shows that the granular temperature in the two configurations begins to differ at the critical volume fraction, reinforcing the idea that $\nu_{c}$ is representative of both shear jamming and the transition to an erodible 
bed. In the latter, the presence of compressible, collisional, bounding regions permits far greater fluctuations in the particle velocity than in the pressure-imposed simple shearing case, consistent with the idea that the contact chains are more prone to buckling and the particles experience more intense rearrangements.

In conclusion, we have employed discrete element simulations of steady flows, in which frictional spheres interact through linear springs and dashpots, to investigate the physics of erodible beds. These are regions in which the solid volume fraction exceeds a critical value for the development of a persistent network of chains that are in contact with compressible, collisional flows. The continuous breaking and forming of chains at their interface with the flow causes strong temporal fluctuations in the coordination number, and this permits the identification of the critical volume fraction with that at shear jamming in simple shearing.

Unlike in shear-jammed states, the contact network in erodible beds has a lower coordination number than that required for mechanical stability. It is the action of a compressible, collisional flow that buckles the chains and creates a fragile contact network that is far more agitated than shear-jammed, granular assemblies at the same value of solid volume fraction. This explains why erodible beds creep slowly, but easily, even under mild shearing $[5,6]$. This description is consistent with the distinction between fragile and shear-jammed states in anisotropic compression of frictional packings of disks based on the percolation of the contact network along the compressive direction or along both compressive and dilational directions [19].

We have indicated that the behaviour of the erodible bed is universal: if the particles are sufficiently rigid, the pressure in the erodible bed is rate-independent and proportional to the particle stiffness -another indirect proof of the existence of a persistent contact network. Shear jammed states also manifest rate-independence, but the effective stiffness is roughly three orders of magnitude larger than that in the erodible beds. The much weaker bulk stiffness of the contact network in the erodible bed, with respect to the shear-jammed state, might have important implications, for example, in those geophysical applications in which granular assemblies are subjected to small strains and behaves elastically [26]. It might also have strong implications in the problem of particle erosion, at least in those cases where the latter is driven by pressure perturbations propagating in an erodible bed [27]. We have noted that the coordination number in almost the entire flow in contact with the erodible bed is less than unity, indicating that collisions are binary and instantaneous. Hence, the largely accepted idea that models of dense granular flows must incorporate enduring and multiple contacts between the particles [28, 29] has little physical justification.

Future work shall focus on the influence of the contact model on our findings on the behaviour of erodible beds; although it is known that employing true Hertzian, rather than linear contacts has little effect on sufficiently rigid particles in the collisional regime, in which the contact duration is much less than the time of free flight, and in the shear-jammed regime [16]. Also, the physical explanation, based on first principles, of the relatively small stiffness of the contact network in erodible beds remains an open issue. Finally, the study of pressure-imposed shear flows in the presence of lateral confinement, i.e., an intermediate configuration between inclined, free surface and pressure-imposed shear flows [30,31], might provide further insights on the possible transition from a fragile to a shear-jammed contact network.

[1] Meiburg E, Kneller B. Turbidity Currents and Their Deposits. Ann Rev Fluid Mech. 2010;42:135-156.

[2] Valance A, Rasmussen KR, El Moctar AO, Dupont P. The physics of Aeolian sand transport. Comptes Rendus Physique. 2015;16(1):105-117.

[3] Frey P, Church M. How river beds move. Science (New York, NY). 2009 sep;325(5947):1509-10. Available from: http://www.ncbi.nlm.nih.gov/pubmed/19762634.

[4] Iverson RM, Reid ME, Logan M, LaHusen RG, Godt JW, Griswold JP. Positive feedback and momentum growth during debris-flow entrainment of wet bed sediment. Nature Geoscience. 2010 dec;4(2):116-121. Available from: http://www.nature.com/doifinder/ 10.1038/ngeo1040.

[5] Komatsu T, Inagaki S, Nakagawa N, Nasuno S. Creep Motion in a Granular Pile Exhibiting Steady Surface Flow. Physical Review Letters. 2001 feb;86(9):17571760. Available from: http://link.aps.org/doi/10. 1103/PhysRevLett.86.1757.

[6] Richard P, Valance A, Métayer JF, Sanchez P, Crassous J, Louge M, Delannay R. Rheology of Confined Granular Flows: Scale Invariance, Glass Transition, and Friction Weakening. Physical Review Letters. 2008 dec;101(24):248002. Available from: http://link.aps . org/doi/10.1103/PhysRevLett.101.248002.

[7] Fraccarollo L, Capart H. Riemann wave description of erosional dam-break flows. Journal of Fluid Mechanics. 2002 jul;461:183-228. Available from: http://www . journals . cambridge .org/abstract $\left\{\backslash_{\text {_ }}\right.$ \}S0022112002008455.

[8] Iverson RM. Elementary theory of bed-sediment entrainment by debris flows and avalanches. Journal of Geophysical Research. 2012;117(F3):1-17. Available from: http://www.agu.org/pubs/crossref/2012/ 2011 JF002189. shtml.

[9] Lees AW, Edwards SF. The computer study of transport processes under extreme conditions. J Phys C: Solid State Phys. 1972;5:1921-1929.

[10] Chialvo S, Sun J, Sundaresan S. Bridging the rheology of granular flows in three regimes. Physical Review E. 2012;85(2):021305. Available from: http://link.aps . org/doi/10.1103/PhysRevE.85.021305.

[11] Berzi D, Jenkins JT. Steady shearing flows of deformable, 
inelastic spheres. Soft Matter. 2015;11(24):47994808. Available from: http://dx.doi.org/10.1039/ C5SM00337G.

[12] Taberlet N, Richard P, Valance A, Losert W, Pasini JM, Jenkins JT, et al. Superstable Granular Heap in a Thin Channel. Phys Rev Lett. 2003;91:264301.

[13] Jop P, Forterre Y, Pouliquen O. Crucial role of side walls for granular surface flows: consequences for the rheology. Journal of Fluid Mechanics. 2005;541:167-192. Available from: http://arxiv.org/abs/cond-mat/0503425.

[14] Jenkins JT, Berzi D. Dense inclined flows of inelastic spheres: tests of an extension of kinetic theory. Granular Matter. 2010 apr;12(2):151-158. Available from: http://www. springerlink.com/index/10.1007/ s10035-010-0169-8.

[15] Berzi D, Jenkins JT. Surface flows of inelastic spheres. Physics of Fluids. 2011;23(1):013303. Available from: http://link.aip.org/link/PHFLE6/v23/ $i 1 / \mathrm{p} 013303 / \mathrm{s} 1\{\backslash \&\}$ Agg=doi.

[16] Sun J, Sundaresan S. A constitutive model with microstructure evolution for flow of rate-independent granular materials. Journal of Fluid Mechanics. 2011 jul;682:590-616. Available from: http://www.journals . cambridge.org/abstract\{\_\}S0022112011002515.

[17] Vescovi D, Luding S. Merging fluid and solid granular behavior. Soft Matter. 2016;12:8616-8628.

[18] Vescovi D, Berzi D, di Prisco CG. Fluid solid transition in unsteady, homogeneous, granular shear flows. Granular Matter. 2018;20:27.

[19] Bi D, Zhang J, Chakraborty B, Behringer RP. Jamming by shear. Nature. 2011;480(7377):355-358. Available from: http://dx.doi.org/10.1038/nature10667.

[20] Berzi D, Jenkins JT, Richard P. Extended Kinetic Theory for collisional shearing over and within an inclined, erodible bed. J Fluid Mech. 2019;p. under review.

[21] Zhang Q, Kamrin K. Microscopic Description of the Granular Fluidity Field in Nonlocal Flow Modeling. Physical Review Letters. 2017;118:058001.

[22] Berzi D, Vescovi D. Different singularities in the functions of extended kinetic theory at the origin of the yield stress in granular flows. Physics of Fluids. 2015;27(1):013302.

[23] Torquato S. Nearest-neighbor statistics for packings of hard spheres and disks. Physical Review E. 1995;51(4):3170-3182. Available from: http://pre.aps . org/abstract/PRE/v51/i4/p3170 $\left.\backslash \backslash_{-}\right\} 1$.

[24] Weinhart T, Hartkamp R, Thornton AR, Luding S. Coarse-grained local and objective continuum description of three-dimensional granular flows down an inclined surface. Physics of Fluids. 2013;25(7):070605.

[25] Zhang Q, Kamrin K. personal communication. personal communication. 2019;.

[26] Kruyt NP. Micromechanical study of elastic moduli of three-dimensional granular assemblies. Int J Sol Struct. 2014;51:2336-2344.

[27] Jenkins JT, Berzi D. Erosion and deposition in depthaveraged models of dense, dry, inclined, granular flows. Phys Rev E. 2016;94:052904.

[28] Forterre Y, Pouliquen O. Flows of Dense Granular Media. Annual Review of Fluid Mechanics. 2008 jan;40(1):124. Available from: http://www.annualreviews.org/ doi/abs/10.1146/annurev.fluid.40.111406.102142.

[29] Ouda M, Toorman E. Development of a new multiphase sediment transport model for free surface flows. Int $\mathrm{J}$
Multiphase Flows. 2019;117:81-102.

[30] Artoni R, Richard P. Effective Wall Friction in WallBounded 3D Dense Granular Flows. Physical Review Letters. 2015;115(15):158001. Available from: http:// link.aps.org/doi/10.1103/PhysRevLett.115.158001.

[31] Artoni R, Soligo A, Paul JM, Richard P. Shear localization and wall friction in confined dense granular flows. J Fluid Mech. 2018;849:395-418. 